\title{
Research on Automotive Outside Airbag System Based on Pyroelectric Infrared Detector
}

\author{
Yanhui Fan ${ }^{1}$, Lingyun $\mathrm{Xiao}^{2}$, Wenhao $\mathrm{Hu}^{3}$, Haiyun $\mathrm{Sun}^{4, *}$ and Rui Cheng ${ }^{5}$ \\ ${ }^{1}$ Shandong Jiao Tong University, No. 5001 Haitang Road, University Science and Technology Park, Changqing District, Jinan \\ City, Shandong Province, China \\ ${ }^{2}$ AQSIQ Defective Product Administrative Center, Amway gardon B-10F, No.66 Anli Road, Chaoyang District, Beijing, China \\ ${ }^{3}$ AQSIQ Defective Product Administrative Center, Amway gardon B-10F, No.66 Anli Road, Chaoyang District, Beijing, China \\ ${ }^{4}$ Shandong Jiao Tong University, No. 5001 Haitang Road, University Science and Technology Park, Changqing District, Jinan \\ City, Shandong Province, China \\ ${ }^{5}$ Shandong Jiao Tong University, No. 5001 Haitang Road, University Science and Technology Park, Changqing District, Jinan \\ City, Shandong Province, China \\ ${ }^{*}$ Corresponding author
}

\begin{abstract}
A new automotive outside airbag system based on operating characteristics of pyroelectric infrared detector is designed. Then, the working principle of airbag system is analyzed, and its effectiveness is verified by PC-CRASH simulation software. The new automotive outside airbag system can make it possible that the airbag system is not exploded when the automotive collide with automotive or fixation, which can save the cost of automotive and be achieve increased popularity.
\end{abstract}

Keywords-pyroelectric infrared detectors; automotive outside airbag system; simulation; effectiveness

\section{INTRODUCTION}

China is a country with typical mixed modes of transportation in the world, causing serious damages to outside people of the vehicle. Statistics show that: the number of deaths of pedestrians, non-motorized drivers and occupants in traffic accidents has exceeded $50 \%$ in China. The in-depth researches on the collision protection system for people outside of the automobile is carried out all over the world. For example, Germany developed a new sensor for accurately detecting pedestrian collisions in automobiles in 2011[1];Volvo Car Corporation applied pedestrian airbags to Volvo V40 vehicles in 2012[2].The R\&D(research and development) and application of these safety technologies have provided a good collision protection effect for the people outside the vehicle.

Based on the characteristics of pyroelectric infrared detectors, the paper designs and develops a new type of automotive outside airbag system, and uses PC-CRASH to verify the effectiveness of the airbag system. The automotive outside airbag system designed and developed in this paper has the advantage of not inflating the airbag when the car collide with cars or fixtures. This technology saves the cost of car use and deserves further promotion.

\section{Analysis of Road Use Vulnerable People COLLISION KINEMATICS}

According to the kinematic analysis of the collision accident between the automobile and the outside of the vehicle, the collision process can be divided into three phases. The first stage: pedestrians, two-wheeled cyclist's legs or two wheelers come into contact with the front bumper of the car (first collision); The second stage, the upper body of the pedestrian/cyclist collide with car bonnet, or the head collide with the car front windshield or A-pillars on both sides (second collision); The third stage, Pedestrians and cyclists vacate and fall on the ground (third collision). The existing research data shows that the second collision and the third collision are the main causes of fatal injury to the people outside the vehicle in the collision accident between the car and the outside people of the vehicle. Therefore, designing and developing an effective outside airbag system is an important technical means of improveing the protection effect of vulnerable road user.

\section{INTRODUCTION OF PYROELECTRIC INFRARED DETECTORS}

Pyroelectric infrared detectors use the thermal radiation of infrared radiation to cause changes in the temperature of the components themselves and output alternating signals. The human body has a constant body temperature of around $37^{\circ} \mathrm{C}$, so it emits infrared light with a wavelength of about $10 \mu \mathrm{m}$. The pyroelectric infrared detector works by detecting infrared radiation emitted from the human body. Compared with photo detectors, the detection rate of pyroelectric infrared detectors has higher stability and anti-interference, and with the increase of operating temperature, its detection rate is more sensitive[3]. Because the car is always facing a complex and changeable environment during the driving process, and the temperature distribution of the car body itself is uneven. Taking all the above factors into considerations, we choose the pyroelectric infrared detector as the object of study.

In order to further improve the accuracy of pyroelectric infrared detectors and avoid burst errors of automotive outside airbags, we use schematic Figure 1 to determine the horizontal sensing angle of the Fresnel lens. 


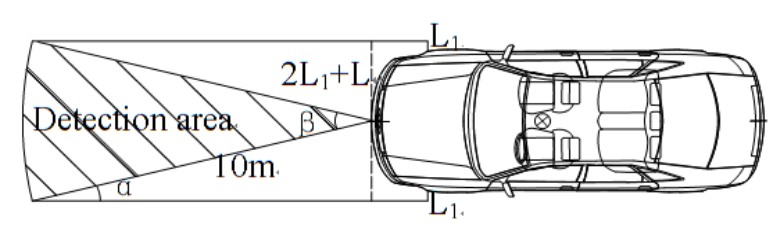

FIGURE I. FRESNEL LENS'S HORIZONTAL INDUCTION ANGLE

For example, with the Volvo V40, the detector is installed directly in front of the car's head. Considering the situation where the car is struck on both sides of the pedestrian, the lateral sides of the car body are widened in the horizontal detection range, as in (1).

$$
\frac{1}{2} \alpha=\beta=\arcsin \frac{2 \mathrm{~L}_{1}+\mathrm{L}}{20}
$$

In formula (1): $\mathrm{L}$ is the vehicle width of the Volvo V40, $1.802 \mathrm{~m} ; \mathrm{L}_{1}$ is widened on both sides of the car body, $0.3 \mathrm{~m}$. Therefore, the horizontal sensing angle of the Fresnel lens is $14^{\circ}$.

\section{A. Pyroelectric Infrared Detector Operating Mode}

When the car is driving at a speed higher than $20 \mathrm{~km} / \mathrm{h}$, the pyroelectric infrared detector installed at the head of the car will continuously receive infrared rays emitted from external objects. Once the human body infrared is detected within $10 \mathrm{~m}$, pedestrian airbags will be immediately bursted through the ECU. On the contrary, the pedestrian airbags will not be bursted. The workflow is shown in Figure 2.

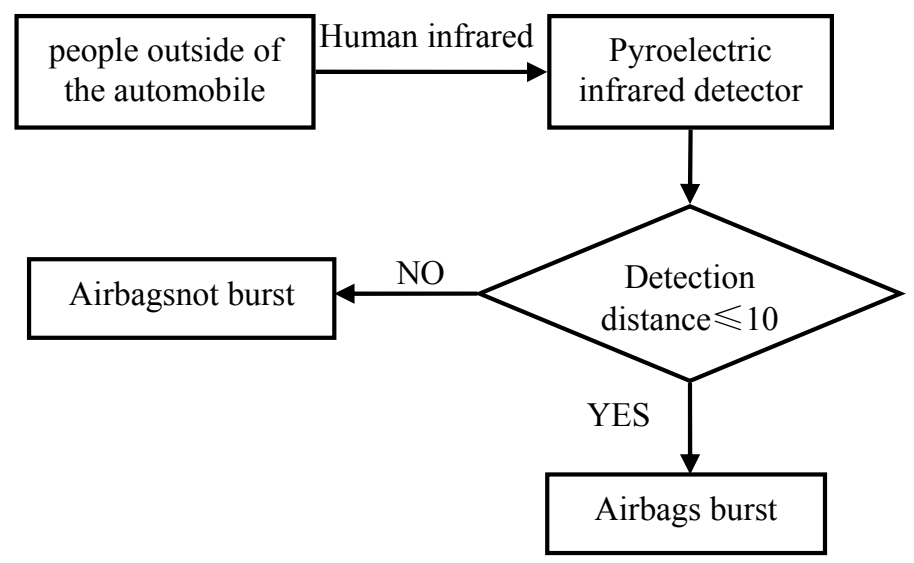

FIGURE II. WORKFLOW CHART

\section{TIME FEASIBILITY OF PYROELECTRIC INFRARED DETECTORS}

Currently, pedestrian airbags include front airbags and hood airbags. In order to effectively reduce the damage caused to the person outside of the vehicle by first collision or second collision, it is necessary to ensure that the front bumper airbag is deployed before the collision, and that the windshield or the front A-pillar airbags are deployed. This requires that pedestrian airbag system equipped with pyroelectric infrared detectors be time-efficient. That is, the working time of the pedestrian airbag system is to be less than the time used before the first collision(TTFC), and less than the time used before the second collision(TTSC).

The TTFC is defined as the time it takes for the detector to detect the body until the first collision between the body and the car. The formula is expressed as (2):

$$
\mathrm{TTFC}=\frac{\mathrm{L}}{\mathrm{V}}
$$

In formula (2): $\mathrm{L}$ is the effective detection distance of the pyroelectric infrared detector, it is about $10 \mathrm{~m}$; $\mathrm{V}$ is the speed of the car.

The TTSC is defined as the time it takes for the detector to detect the body until the second collision between the body and the car, usually TTFC $<$ TTSC.

The Figure 3 shows a schematic diagram of signal transmission from a pyroelectric infrared detector detecting human infrared to pedestrian airbag burst.

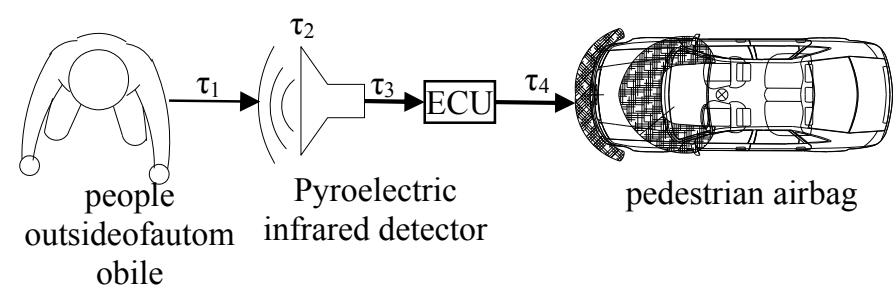

FIGURE III. SIGNAL TRANSMISSION DIAGRAM

In Figure 3: $\tau_{1}$ is the propagation time of the human body's infrared arrival detector; $\tau_{2}$ is the time when the pyroelectric infrared detector receives infrared light and converts it into an electrical signal; $\tau_{3}$ is the time when the ECU receives the electric signal from the pyroelectric infrared detector and transmits it to the pedestrian airbag system; $\tau_{4}$ is the deployment time of pedestrian airbag.

Because the infrared transmission speed is about $3 \times 10^{8} \mathrm{~m} / \mathrm{s}$.Therefore, $\tau_{1}$ can be neglected within a short distance. By consulting relevant data, we know that the response time of pyroelectric infrared detectors currently produced is basically the same as the response time of photoelectric detectors[4]. The photodetector response time is $0.32 \mathrm{~ms}$ to $1.54 \mathrm{~ms}[5]$, in the paper, $\tau_{2}$ takes $1.54 \mathrm{~ms}$;ECU response time is takes $20 \mathrm{~ms}$;Pedestrian airbag deployment time is $50 \mathrm{~ms}$ to $75 \mathrm{~ms}[6]$,in the paper, $\tau_{4}$ takes $75 \mathrm{~ms}$. Then the working time of the pedestrian airbag system is about $96.54 \mathrm{~ms}$.

\section{Feasible Design Plan}

Through investigating numerous traffic accident cases, it was found that the types of collisions between cars and people outside of the vehicle were mostly side collision. That is, the 
head of the car collided with the side of a pedestrian or a twowheeler. The working condition of the pedestrian airbag system is that the vehicle speed is higher than $20 \mathrm{~km} / \mathrm{h}$. Therefore, when using the Pc-Crash software simulation, the accidental collision mode is set to side collision, and the car does not adopt braking and steering measures. The vehicle speed is set as $20 \mathrm{~km} / \mathrm{h}, 30 \mathrm{~km} / \mathrm{h}, 40 \mathrm{~km} / \mathrm{h}, 50 \mathrm{~km} / \mathrm{h}, 60 \mathrm{~km} / \mathrm{h}, 70 \mathrm{~km} / \mathrm{h}, 80 \mathrm{~km} / \mathrm{h}$ Pedestrian walking speed is set to $5 \mathrm{~km} / \mathrm{h}$; The speed of the twowheeler is set to $15 \mathrm{~km} / \mathrm{h}$.

\section{PC-CRASH SIMULATION}

\section{A. Car-Pedestrian Model}

First, the Ford-Volvo 40 rigid body model produced by Volvo is transferred to the PC-CRASH simulation software. Second, the article sets the relevant parameters of the car. Then the multi-rigid model of pedestrians is transferred and the mass is set to $75 \mathrm{~kg}$ and the walking speed is $5 \mathrm{~km} / \mathrm{h}$. The initial relative distance of the participants is $10 \mathrm{~m}$, and the road adhesion coefficient is 0.7 . The adjusted model is shown in Figure 4.

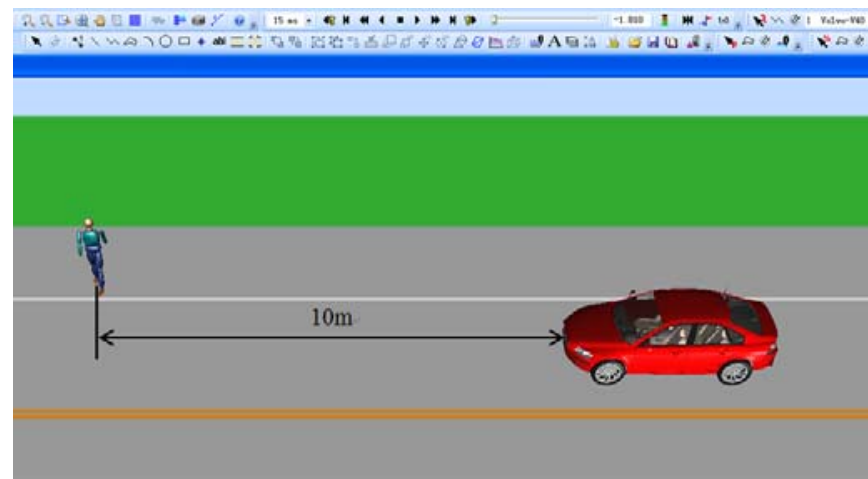

FIGURE IV. PRE-COLLISION INITIAL POSITION AND TIME

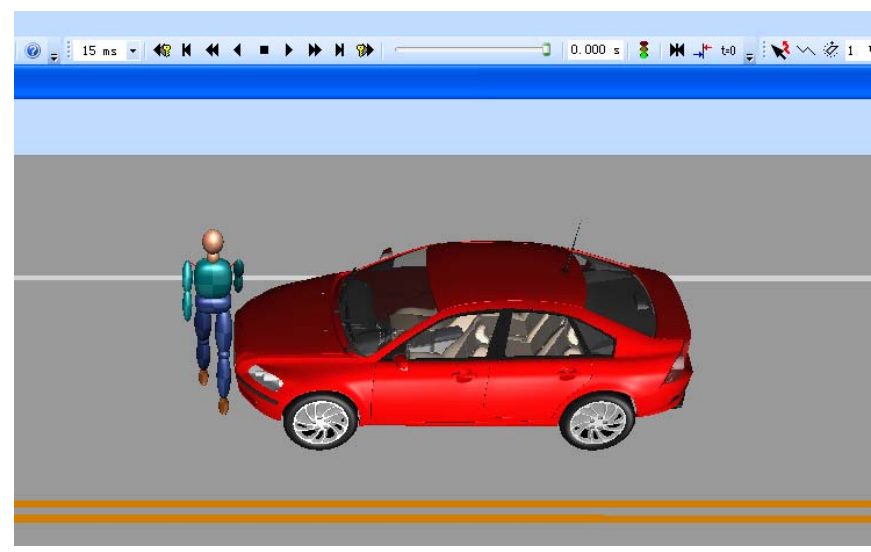

FIGURE V. FIRST COLLISION START TIME

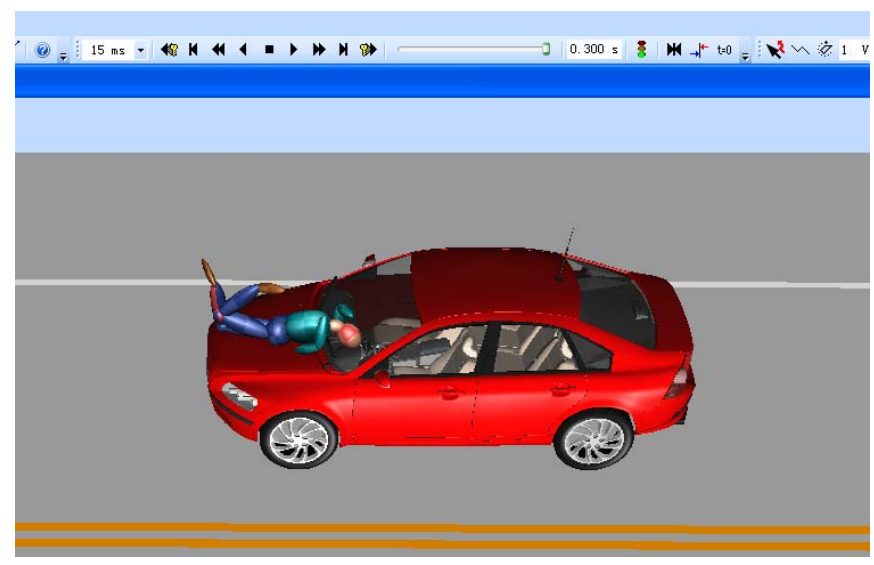

FIGURE VI. THE SECOND COLLISION START TIME

The conclusion is that: $T$ TFC $1=0 \mathrm{~s}-(-1.81 \mathrm{~s})=1810 \mathrm{~ms}$; TTSC $1=0.3 \mathrm{~s}-(-1.81 \mathrm{~s})=2110 \mathrm{~ms}$.

Set the vehicle speed to $V_{i}(i=1, \ldots, 6,7)$ and get the simulation results as shown in Table 1 below:

TABLE I. SPEED-TIME

\begin{tabular}{|c|c|c|c|}
\hline Speed $(\mathrm{km} / \mathrm{h})$ & TTFC $(\mathrm{ms})$ & TTSC $(\mathrm{ms})$ & $\Gamma(\mathrm{ms})$ \\
\hline 20 & 1810 & 2110 & 96.54 \\
\hline 30 & 1215 & 1425 & 96.54 \\
\hline 40 & 915 & 1080 & 96.54 \\
\hline 50 & 730 & 865 & 96.54 \\
\hline 60 & 610 & 715 & 96.54 \\
\hline 70 & 525 & 623 & 96.54 \\
\hline 80 & 465 & 540 & 96.54 \\
\hline
\end{tabular}

The result is plotted as a graph as shown in Figure 7.

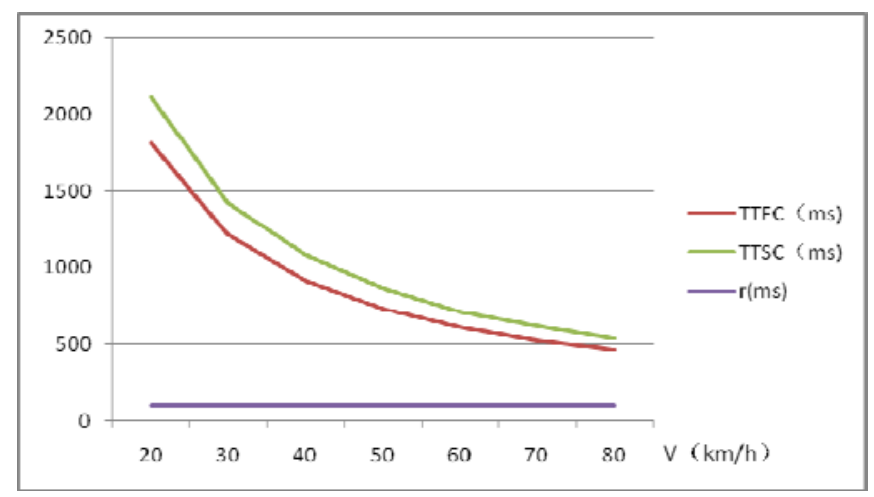

FIGURE VII. SPEED-COLLISION TIME LINE CHART

\section{B. Car - Two-Wheeler Model}

The multi-rigid model of the two-wheeled vehicle is transferred to the PC-CRASH simulation software. The driving speed is set to $15 \mathrm{~km} / \mathrm{h}$ and the mass is $127 \mathrm{~kg}$. According to the car and pedestrian simulation steps, the simulation results are shown in Table 2 below: 
TABLE II. SPEED-TIME

\begin{tabular}{|c|c|c|c|}
\hline $\begin{array}{c}\text { Speed } \\
(\mathrm{km} / \mathrm{h})\end{array}$ & TTFC $(\mathrm{ms})$ & TTSC(ms $)$ & $\Gamma(\mathrm{ms})$ \\
\hline 20 & 1810 & 2080 & 96.54 \\
\hline 30 & 1215 & 1440 & 96.54 \\
\hline 40 & 915 & 1080 & 96.54 \\
\hline 50 & 730 & 865 & 96.54 \\
\hline 60 & 610 & 730 & 96.54 \\
\hline 70 & 525 & 625 & 96.54 \\
\hline 80 & 465 & 550 & 96.54 \\
\hline
\end{tabular}

The result is plotted as a graph as shown in Figure 8.

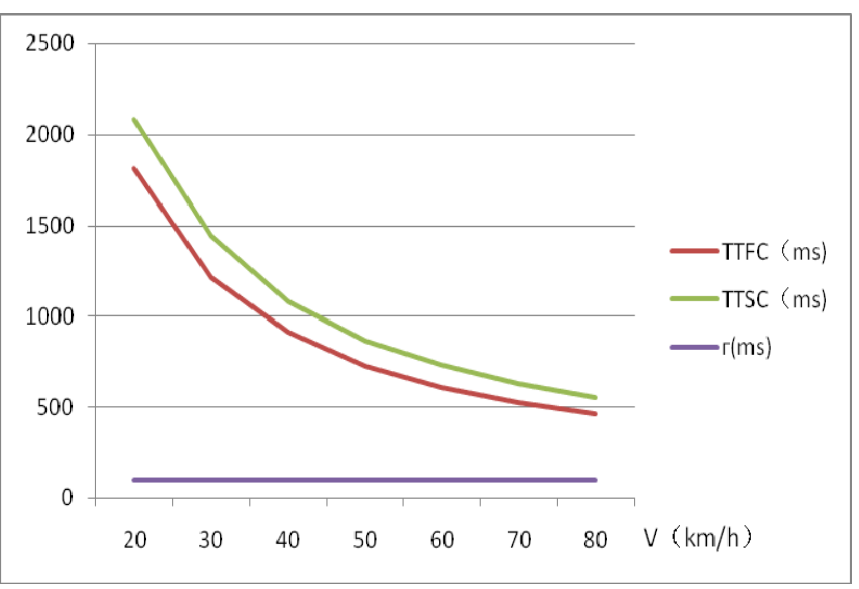

FIGURE VIII. SPEED-COLLISION TIME LINE CHART

As is seen from Figure 7 and Figure 8,when a car collides with pedestrian or two-wheeler and the vehicle speed is higher than $20 \mathrm{~km} / \mathrm{h}$, ris less than TTFC and TTFC is less than TTSC.As a result, pedestrian airbag system based on pyroelectric infrared detector has time feasibility.

\section{CONCLUSION}

The paper verifies the feasibility of pedestrian airbag system based on pyroelectric infrared detectors by Pc-Crash software. The system can selectively open pedestrian airbags, which not only saves costs, but also reduces the impact of airbag error burst on the driver's operation. Although the actual feasibility of the system has not been further verified, but its time feasibility has been fully demonstrated. In addition, the specific installation position of the pyroelectric infrared detector and the selection of performance parameters deserve further study.

\section{ACKNOWLEDGMENT}

This project is supported by Outstanding Middle and Young Scientists Research Award Fund of Shandong Province (BS2011SF014), Science and Technology Project of Higher School of Shandong Province (J11LG17), Natural Science Foundation of Shandong Province (ZR2010EL023) and National Automobile Accident In-depth Investigation System. This project would not have been possibly accomplished without these funds support.

\section{REFERENCES}

[1] Di Lu, "Germany has developed a new sensor that accurately detects the collision of cars and pedestrians"[N],Electronic Engineering World, July 252011.

[2] Xianfu Yang,"Analysis of VOLVO V40 Car Pedestrian Airbag System and Its Maintenance"[J],Automotive Appliances,2015, (1): 19-21.

[3] Gehong Zeng,"Infrared photon detector and thermal detector performance analysis"'J].Infrared technology,2011,33(9):497-500.

[4] Yu Han, "Research on Pyroelectric Infrared Detector Based on Wafer Bonding Technology"[D].Measuring and Testing Technologies and Instruments,North University of China,2011.

[5] Lianjia Wang, "Response time analysis of AlGaN deep ultraviolet photodetectors"[J],Sichuan Journal of Ordnance, 2010, 31(11): 137-140.

[6] K. Elissa, "Discussion on Automobile Airbag Technology Outside Vehicles"[J].Automotive Engineer,2010, (9): 17-20. 